Revista Chilena
De Pediatría

www.revistachilenadepediatria.cl

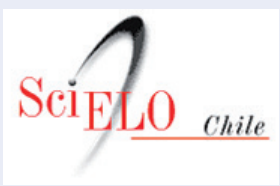

www.scielo.cl

\title{
Cánula nasal de alto flujo en la bronquiolitis aguda: la evidencia sigue pendiente
}

\author{
High-flow nasal cannula therapy in acute bronchiolitis: \\ the evidence is still pending
}

\section{Raúl Bustos B. ${ }^{\mathrm{a}}$}

aMedicina Intensiva Pediátrica, Hospital Guillermo Grant Benavente y Clínica Sanatorio Alemán, Concepción

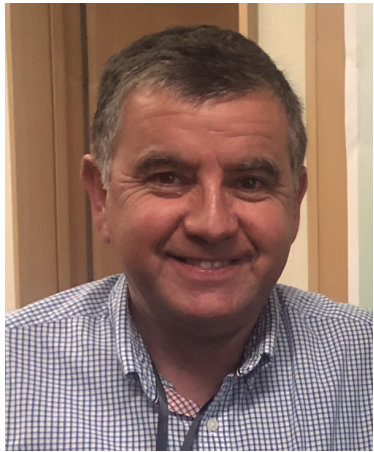
Chile

La bronquiolitis es una infección frecuente de la via respiratoria baja caracterizada por inflamación aguda de la vía aérea pequeña, y representa la primera causa de hospitalización en niños durante el primer año de vida. En Estados Unidos se estima que 100 mil niños serán hospitalizados al año con gastos asociados a 1,73 billones de dólares ${ }^{1}$.

Múltiples intervenciones farmacológicas que han intentado cambiar el curso clínico de la bronquiolitis aguda, han fallado en demostrar algún beneficio, lo que ha llevado a la publicación de guías clínicas nacionales de tratamiento en diferentes latitudes. Así, guías de Australia, Inglaterra y Estados Unidos enfatizan que sólo la administración de oxígeno y la hidratación como efectivas y no recomiendan el uso de agentes farmacológicos o exámenes adicionales. Sin embargo, a pesar de la diseminación de estas guías, aún existe un uso importante de terapias no recomendadas y variabilidad en el tratamiento de los niños que se hospitalizan por bronquiolitis. Un estudio multicéntrico internacional en 38 unidades de emergencia, demostró que más de un 30\% de niños de menos de 1 año hospitalizados por bronquiolitis, aún reciben terapias farmacológicas y exámenes adicionales no recomendados².

En países desarrollados, aproximadamente uno de ocho niños hospitalizados por bronquiolitis van a requerir ingreso a unidades de cuidado intensivo (UCI) por insuficiencia respiratoria e hipoxemia. La CPAP (continous positive airways pressure), ha sido tradicionalmente empleada como soporte respiratorio no invasivo de primera línea en la bronquiolitis para prevenir la intubación y el uso de ventilación invasiva. Recientemente, una modalidad alternativa de soporte respiratorio no invasivo, la cánula nasal de alto flujo (CNAF) ha adquirido popularidad por su facilidad de uso y buena tolerancia. A través de distintos mecanismos, entre los que se incluyen, reducción en la resistencia de la vía aérea, reducción del espacio muerto por washout del espacio nasofaríngeo y un efecto CPAP, la CNAF ha demostrado reducir el trabajo respiratorio y mejorar la oxigenación y ventilación en niños ${ }^{3}$. Un estudio observacional realizado en más de nueve mil niños con bronquiolitis ingresados a UCI, demostró un aumento significativo en el uso de la CNAF. Hasta un $70 \%$ de los ingresos fueron tratados con esta modalidad ventilatoria ${ }^{4}$. Sin embargo, en una época de medicina basada en la evidencia, llama la atención la popularidad que ha adquirido esta técnica con la escasa evidencia de su efectividad.

Un estudio observacional de diseño antes y después

Correspondencia:

Raúl Bustos B.

rbustos@sanatorioaleman.cl 
de una guía de implementación de CNAF en un servicio de pediatría, que incluyo 1.937 niños, no logró demostrar una disminución en los días totales de hospitalización, días de estadía e ingreso en UCI y tasas de intubación ${ }^{5}$.

El estudio aleatorizado y controlado PARIS (Pediatric Acute Respiratory Intervention Study), que comparó el uso de la CNAF con la cánula de oxigeno convencional, realizado en cerca de mil quinientos niños con bronquiolitis en salas de urgencia y servicios de pediatría en Australia y Nueva Zelanda, si bien, concluyo un significativa disminución en la intensificación del tratamiento en los niños que recibieron CNAF comparado al grupo con cánula estándar, no demostró diferencias significativas entre los grupos en la duración de los días de oxígeno, hospitalización y estadía en la UCI. Tampoco se observaron diferencias en los ingresos a UCI e intubación endotraqueal entre ambos grupos. Por otro lado, más de tres cuartos de los niños manejados con cánula standard no necesitaron intensificación en su tratamiento. También es sorprendente que los niños que recibieron terapia con la CNAF, tenían una frecuencia respiratoria significativamente más elevada $(62,6 \pm 12,4 \mathrm{rpm})$ que aquellos en el grupo de tratamiento estándar $(54,6 \pm 15,2 \mathrm{rpm})$ al momento del escalamiento en el tratamiento ${ }^{6}$.

Otro estudio de similar diseño de un centro, que incluyó doscientos niños, no logro demostrar una disminución de la duración del oxígeno en los pacientes asignados al tratamiento con CNAF comparados a aquellos tratados con cánula tradicional. La estadía hospitalaria e ingreso a UCI fueron similares en ambos grupos ${ }^{7}$.

Milesi al comparar la CNAF con la CPAP en ni- ños con bronquiolitis ingresados en UCI ,concluyo que CNAF no demostró ventajas respecto a la CPAP, el fracaso en CNAF fue de $51 \%$ comparado al $31 \%$ de fracasos en CPAP. En otras palabras, la CPAP resultó ser más eficiente que la CNAF en el soporte respiratorio inicial en niños con bronquiolitis hospitalizados en UCI. Confirmando que hasta el día de hoy, la CNAF no ha demostrado ser equivalente o superior a la CPAP8.

La historia del tratamiento de la bronquiolitis, particularmente en los pacientes hospitalizados, es de la incorporación rápida de terapias, antes que una evidencia sólida avale su uso. El caso de la solución salina hipertónica es un ejemplo categórico, si bien, su uso en de ensayos controlados demostró beneficios en términos de reducción en la hospitalización y estadía hospitalaria, estos hallazgos han sido desmentidos posteriormente en meta análisis convencionales y avanzados?.

Los pediatras que trabajamos al cuidado de niños críticamente enfermos, hemos pasado décadas adoptando de manera entusiasta nuevas opciones de tratamiento para nuestros pacientes con bronquiolitis, sobre todo si estas nuevas tecnologías tienen un sustento fisiopatológico. Una vez que esta tecnología ha sido adoptada -a pesar que la evidencia diga lo contrariose hace más difícil abandonar su uso. Probablemente, este será también el camino de la CNAF en la bronquiolitis aguda.

\section{Conflicto de intereses}

El autor declara no tener conflicto de intereses.

\section{Referencias}

1. Hasegawa K, Tsugawa Y, Brown DF, Mansbach JM, Camargo CA Jr. Trends in bronchiolitis hospitalizations in the United States, 2000-2009. Pediatrics. 2013;132:28-36.

2. Schuh S Babl FE Dalziel SR et al. Practice variation in acute bronchiolitis: A Pediatric emergency Research Network Study Pediatrics 2017;140:e20170842.

3. Lee M Jr, Nagler J. High-flow nasal cannula therapy beyond the perinatal period. Curr Opin Pediatr. 2017;29: 291-6.

4. Schlapbach LJ, Stanley L, Gelbart B, et al.
Burden of disease and change in practice in critically ill infants with bronchiolitis Eur Respir J 2017;49:1601-48.

5. Riese J, Porter T, Fierce J, Riese A, Richardson T, Alverson BK. Clinical Outcomes of Bronchiolitis After Implementation of a General Ward High Flow Nasal Cannula Guideline. Hosp Pediatr. 2017;7:197-203.

6. Franklin D, Babl FE, Schlapbach LJ, et al. A Randomized Trial of HighFlow Oxygen Therapy in Infants with Bronchiolitis. N Engl J Med. 2018;22;378:1121-31.

7. Kepreotes E, Whitehead B,Attila J, et al. High Flow warm humidified oxygen versus standard low flow nasal cannula oxygen for moderate bronquiolitis: an open phase 4 Randomised controlled trial Lancet 2017;369:930-9.

8. Milési C, Essouri S, Pouyau R, et al High flow nasal cannula versus nasal continuous positive airway pressure for the initial respiratory management of acute viral bronchiolitis in young infants :a multicenter randomized controlled trial. Intensive Care Med 2017;43: 209-16.

9. Harrison W, Angoulvant F, House S, Gajdos V, Ralston S. Hypertonic saline in bronquiolitis and type I error :A trial sequential analysis Pediatrics 2018;142 . 